\title{
Gadolinium Induced Acute Kidney Injury and Nephrogenic Systemic Fibrosis
}

\author{
Khurshid A. Banday, M.D., Irfan Robbani, M.D., Nasir Ahmad Choh, M.D.,' Farhana Siraj, M.D., \\ Fayaz ul Haque, M.D., I.A. Wani, D.M., M.M. Wani, D.M. \\ Departments of Nephrology \&'Radiology, Sher-i-Kashmir Institute of Medical Sciences, Soura, Srinagar, Kashmir.
}

\section{A B S T R A C T}

\begin{abstract}
Use of contrast agents like gadolinium in MR imaging studies has considerably increased. Although safe in patients with normal kidney function, administration of these agents in people with renal dysfunction can result in many clinical problems that the nephrologists should be familiar with. This brief review discusses these iatrogenic problems that can be induced by contrast agents like gadolinium. JMS 2012;15(2):166-69
\end{abstract}

Key words: Gadolinium, MRI, contrast agents, renal function test, nephrotoxicity

Magnetic resonance imaging (MRI) scans are significantly enhanced by use of gadolinium based contrast (GBC) agents. ${ }^{1}$ Until recently the use of gadolinium was thought to be risk-free compared with alternative contrast agents. ${ }^{2,3}$ Recent studies, however, had raised serious concerns regarding the safety of gadolinium chelates. ${ }^{4}$ Although safe in patients with normal kidney function, administration of these agents in people with renal dysfunction can result in up to three clinical problems that the nephrologists should be familiar with.

First is GBC associated nephrotoxicity (acute kidney injury) particularly in patients with underlying kidney disease. ${ }^{9,10}$ Second and more concerning was the development of nephrogenic systemic fibrosis in patients with advanced kidney failure. ${ }^{11-13}$ Of less significance is a labora-

\section{Correspondence:}

Dr. Khurshid A Banday

Room 2310, Department of Nephrology

Sher-i-Kashmir Institute of Medical Sciences, Soura, 190011 ,

Srinagar, Kashmir.

E-mail: kabanday@gmail.com tory artifact of pseudohypocalcemia. This review with discuss the rise and fall of these iatrogenic complications.

\section{Gadolinium Based Contrast agents (GBC's)}

Gadolinium (GD) is a lanthanide metal with paramagnetic properties. ${ }^{1}$ It is used as intravenous or intra-arterial contrast agent while performing MRI scan. GD in its ionic form is highly toxic; this can be avoided by having GD in chelate form. Chelates are organic molecules which form stable, non-toxic and bio-chemically inert complexes with GD. ${ }^{1,5}$ Gadodiamide (Omniscan), Gadopentetate dimeglumine (Magnevist), Gadobenate dimeglumine (MultiHance) and Gadoteridol (ProHance) are some GD containing chelates commonly used. ${ }^{1}$ Macrocyclic chelates are less toxic as they bind GD more tightly and are less likely to liberate free GD. They are eliminated unchanged by the kidneys with a mean terminal half-life $\left(\mathrm{t}^{1} / 2\right)$ of 1.3 hours. The $\mathrm{t} 1 / 2$ is prolonged to 5.6 hours in patients with moderate renal failure and up to 30 hours in patients with GFR of $<5 \mathrm{ml} / \mathrm{min}^{1,3,4}$ 


\section{Nephrotoxicity (Acute Kidney Injury)}

Since GBC agents have significantly lower viscosity compared to iodinated radio contrast agents and the volume used is 4-11 times less, the nephrotoxicity due to GD was thought to be significantly less. ${ }^{2,3}$ Nephrotoxicity of GBC agents has been studied in experimental animals and minimal elevation of serum creatinine was reported when high doses $\left(>1.0 \mathrm{mmol} / \mathrm{kg}\right.$ ) were used. ${ }^{4}$ Nephrotoxicity of GBC agents has been studied in healthy individuals and in patients with mild to moderate kidney disease ${ }^{6,7,89}$ Several of these studies demonstrated that GBC agents lack significant nephrotoxicity particularly in patients who have normal or minimally elevated $(<2.5 \mathrm{mg} / \mathrm{dl})$ serum creatinine. ${ }^{7.8}$ In these studies GBC associated nephrotoxicity developed in $0-5 \%$ of patients which was less than iodinated contrast (17-40\%).

In contrast to these favorable studies, a number of other studies suggest that GBC's exhibit variable degrees of nephrotoxicity. ${ }^{90}$ GD based contrast agents are reported to induce acute kidney injury (AKI) in a high-risk population group at the usual dose for MRI and MRA examinations. This was noted more frequently in patients with elevated baseline creatinine $\geq 3 \mathrm{mg} / \mathrm{dl}$ ) and in patients with hypertension, diabetes mellitus and sepsis. ${ }^{10}$ In these studies nephrotoxicity developed in $5-50 \%$ of patients which was equal to or greater than that seen with iodinated radio contrast agents. In conclusion majority of studies suggest renal safety but GBC induced nephrotoxicity can develop. Factors which increase the risk of GBC associated nephrotoxicity are advanced CKD, higher doses, and arterial injection.

\section{Nephrogenic Systemic Fibrosis (NSF)}

NSF is a rare syndrome characterized by fibrotic skin, muscle contractures, decreased range of joint motion and organ fibrosis. ${ }^{14,15}$ The first case of NSF was reported in 1997, since then many cases and small cases series have been reported. ${ }^{12-18}$ The largest series from India consists of seven patients of NSF reported from Kolkata. ${ }^{19}$ It was initially recognized as a novel, idiopathic, progressive, scleromyxedema like fibrosing disorder of the skin but soon it was recognized that fibrosing process is not limited to skin but occurs as a systemic condition. ${ }^{15}$ Over last decade multiple reports have revealed an associated between NSF and prior exposure to GBC agents in patient with advanced renal failure. ${ }^{15}$ However no new case of NSF has been reported from USA after 2009. Patient, physician, hospital, product, regulatory and country considerations account for the emergence and disappearance of NSF.

\section{Epidemiology}

NSF has been exclusively reported in patients with renal disease with a glomerular filtration rate (GFR) $<60$ $\mathrm{ml} / \mathrm{min} / 1.73 \mathrm{~m}^{2}$. NSF has been reported in the setting of acute or chronic kidney disease, as well as in either haemo or peritoneal dialysis therapy. ${ }^{16}$ Approximately $90 \%$ of cases have been reported in dialysis patients and $10 \%$ of cases in patients with stage 3 or 4 CKD. ${ }^{12} \mathrm{NSF}$ is an indolent disease with several weeks to months of symptoms prior to diagnosis but can present as rapidly progressive disease often with systemic involvement. ${ }^{16}$ NSF occurs in patients regardless of age, gender or race and has been reported in pediatric patients as young as 8 years old. ${ }^{17,18}$ Fibrosis affecting skin and or systemic organs dominates the clinical picture. ${ }^{20}$

\section{Cutaneous Involvement}

NSF first affects the skin as dermal hardening with tethering to deep dermal tissue, giving a characteristic woody feel. ${ }^{20}$ Additional skin findings are brownish hyper pigmented plagues, brawny indurations, waxy erythematous papules, and subcutaneous nodules. Extremities as well as trunk may be affected but NSF typically presents in lower extremities at calves in a symmetrical fashion. Involvement of upper extremities follows the development of disease in lower extremities. Trunk is involved late in the severe form of the disease whereas face is usually spared. ${ }^{15,20}$

\section{Systemic Involvement}

Systemic fibrosis is an important and a well recognized feature of NSF and can involve skeletal muscles, bones, lungs, pleura, pericardium, myocardium, renal tubules, testes and duramater. ${ }^{15,21}$ NSF can lead to joint contractures due to fibrosis of periarticular structures involvement of shoulders, elbows, wrists and hands causes significant functional disability. Joint contractures of hips, knees and ankles can predispose to falls and factures. Lung involvement leading to fibrosis and diffuse diaphragmatic infiltration leading to respiratory failure is known in NSF.

\section{Etiology}

No definite correlation is yet established but a possible connection with severity of renal failure, history of hyperthyroidism, thrombotic episodes, dependent edema, and vascular procedures has been reported. Many drugs including high dose erythropoietin therapy, past angiotensin converting enzyme inhibitor and sevelamer therapy, has been implicated but not statistically proved. ${ }^{22,23}$ An Austrian study correlated NSF with Gadolinium exposure, renal insufficiency and concurrent acidosis whereas a Danish study calculated a strong link between GD exposure and subsequent onset of NSF in renal insufficiency patients with or without acidosis. ${ }^{14}$

\section{Diagnosis}

Often physical examination and laboratory analysis are adequate to make the diagnosis of NSF in appropriate clinical setting. Non-Gadolinum enhanced MRI may reveal skin thickening and elevated T2 signals in muscles. Skin biopsy is needed in some cases to confirm diagnosis. ${ }^{20}$ 


\section{Histopathology of NSF}

Well described pathological features of NSF include dermal thickness, increased collagen bundles, increased mucin, fibroblast proliferation and spindle shaped fibroblast. Fibrosis can extend into subcutaneous tissue, fascia and even skeletal muscles. Immunolabelling for CD 34 and procollagen I in the spindle cells of NSF may provide a sensitive and specific method of diagnosis. Scanning electron microscopy and inductively coupled mass spectrometry identified GD in biopsies from NSF patients. ${ }^{15,16}$

\section{Differential Diagnosis}

The closest differential diagnosis of NSF is scleromyederma in which skin thickness occur over head, neck, arms and upper trunk while as in NSF characteristic lesional distribution (acral and truncal preferences, facial sparing), raised ESR and CRP lack of inflammatory cells on histology, lack of paraproteinemia and characteristic immunohistochemistry with CD 34 and procollagen I favors the diagnosis of NSF. Systemic sclerosis may be considered as second most important differential diagnosis but serologic finding and histopathology provides the clue. Eosinophilic fasciitis is characterized by peripheral eosinophilia, hypergammaglobinemia and dermal inflammation on skin biopsy and thus differentiating it from NSF. ${ }^{19}$

\section{Treatment}

Physiotherapy is advised to maintain joint mobility. Many pharmacological agents have been tried to improve skin and systemic fibrosis including corticosteroids, thalidomide, cyclophosphamide, mycophenolate mofetil, intravenous immunoglobulin with variable results. Plasma exchange and plasmapheresis has been reported to improve skin fibrosis. Extracorporeal photopheresis has been shown to be more effective than others in improving fibrosis but is very expensive..$^{2426}$

To conclude it is important that the clinicians should be cognizant of GBCA's risk and benefits. NSF fears should not lead to denial of enhanced MR imaging examinations with lower risk stable GBCA's, when clinically appropriate. It is important to identify high-risk patients and closely monitor renal function after administration of GD.

\section{References}

1. Bellin MF. MR contrast agents, the old and the new. Eur J Radiol 2006;60:314-23.

2. Hammer FD, Goffette PP, Malaise J, Mathurin P. Gadolinium dimeglumine: an alternative contrast agent for digital subtraction angiography. Eur Radiol 1999; 9:128-36.

3. Erley CM, Bader BD, Berger ED. Gadolinium based contrast media compared with iodinated media compared with iodinated media for digital subtraction angiography in azotemic patients. Nephrol Diabet Transplant 2004;19: 2526-31.

4. Prince MR, Arnoldus C, Friscoli JK. Nephrotoxicity of high dose gadolinium compared with iodinated contrast.J Magn Reson Imaging 1996; 6: 162-66.

5. Perazella MA. Current status of gadolinium toxicity in patients with kidney diseases. Clin J Am Soc Nephrol 2009; 4: 461-69.

6. Arsenault TM, king BF, Marsh JW JR, Goodman JA, Weaver Al, Wood CP, Ehman RL. Systemic gadolinium toxicity in patients with renal insufficiency and renal failure: retrospective analysis of an initial experience. Mayo Clin Proc 1996; 71: 1150-54.

7. Sam AD, Morasch MD, Collins J, Song G, Chen R, Pereles FS. Safety of gadolinium contrast angiography in patients with chronic renal insufficiency.J Vasc Surg 2003; 38:313-18.

8. Ergun I, Keven K, Uruc I, Ekmekci Y, Canbakan B, Erden I, Karatan O. The safety of gadolinium in patients with stage 3 and 4 renal failure. Nephrol Dial Transplant 2006; 21: 697-700.

9. Chien CC, Wang HY, Wang JJ, Kan WC, Chien TW, et al. Risk of acute kidney injury after exposure to gadolinium-based contrast in patients with renal impairment. Ren Fail 2011;33:758-64.

10. Badero OJ, Schlanger L, Rizk D. Gadolinium nephrotoxicity: case report of a rare entity and review of the literature. Clin Nephrol 2008;70:518-22.

11. Daram SR, Cortese CM, Bastani B. Nephrogenic fibrosing dermopathy/nephrogenic system fibrosis: report of a new case with literature review. Am J Kidney Dis 2005;46: 754-9.

12. Cowper SE, Robin HS, Steinberg SM, Sri LD, Gupta S, Le Boit PE. Scleromyxoedema like cutaneous disease in renal dialysis patients. Lancet 2000; 356:1000-1.

13. Broome DR, Girguis MS, Baron PW, Cottrell AC, Kjellin J, Kirk GA. Gadodiamide associated nephrogenic systemic fibrosis: why radiologists should be concerned. Am J Roentgenol 2007;188: 586-92.

14. Grotmer T. Gadolinium - a specific trigger for the development of nephrogenic fibrosing dermopathy and nephrogenic systemic fibrosis? Nephrol Dial Transplant 2006; 21: 1104-8.

15. Gibson SE, Farver CF, Paryson RA. Multiorgan involvement in nephrogenic fibrosing dermopathy: an autopsy case and review of the literature. Arch Pathol Lab Med 2006;130: 209-12.

16. Mendoza FA, Artelt CM, Sandorfi N, Latinis K, Pieravelazquez S, Jimenez SA. Description of 12 cases of nephrogenic fibrosing dermopathy and review of literature. Semin Arthritis Rheum 2006;35:238-49. 
17. Auron A, Shao L, Warady BA. Nephrogenic fibrosing dermopathy in children. Pediatr Nephrol 2006;21:1307-11.

18. Jan F, Segal JM, Dyer J, LeBoit P, Siegfried E, Frieden IJ. Nephrogenic fibrosing dermopathy: two pediatric cases.J Pediatr 2003;143:678-81.

19. Panda S, Bandyopadhyay D, Taraphder A. Nephrogenic fibrosing dermopathy: A series in a non-western population.J Am Acad Dermatol 2006;54:155-9.

20. Todd DJ, Kagan A, Chibnik L, Kay I. Cutaneous changes of nephrogenic systemic fibrosis predict early mortality and are associated with gadolinium exposure. Arthritis Rheum, In Press.

21. Sainz A, Mandal K, Kradin R, Hedley-Whyte ET. Nephrogenic fibrosing dermatopathy with involvement of the dura matter. Virchows Arch 2006;449:389-91.

22. Swaminathan S, Ahmed I, McCarthy JT, et al.
Nephrogenic fibrosing dermopathy and high dose erythropoietin therapy. Inn Intern Med 2006;145 234-5.

23. Fazeli A, Lio PA, Liu V. Nephrogenic fibrosing dermopathy: are ACE inhibitors the missing link. Arch Dermatol 2004;140:1401.

24. Baron PW, Cantos K, Hillebrand DJ, et al. Nephrogenic fibrosing dermopathy after liver transplantatioin successfully treated with plasmapharesis. Am J Dermatopathol 2003;25 204-9.

25. Chung HJ, Chung KY. Nephrogenic fibrosing dermopathy: response to high dose intravenous immunoglobulin. BrJ Dermatol 2004;150:596-7.

26. Gilliet M, Cozzio A, Burg G, Nestle FO. Successful treatment of three cases of nephrogenic fibrosing dermopathy with extracorporeal photopheresis. $\mathrm{Br} J$ Dermatol 2005;152:531-6. 